\title{
Characteristics of cortical spreading depression and c-Fos expression in transgenic mice having a mutation associated with familial hemiplegic migraine 2
}

Cephalalgia

$0(0) 1-14$

(C) International Headache Society 2020

Article reuse guidelines:

sagepub.com/journals-permissions DOI: $10.1177 / 0333102420929028$ journals.sagepub.com/home/cep @SAGE

\author{
Chunhua Tang',2, Miyuki Unekawa', Mamoru Shibata', \\ Yutaka Tomita', Yoshikane Izawa', Hiroki Sugimoto ${ }^{3}$, \\ Keiko Ikeda ${ }^{3,4}$, Kiyoshi Kawakami ${ }^{3}$, Norihiro Suzuki ${ }^{5}$ and \\ Jin Nakahara'
}

\begin{abstract}
Background: Cortical spreading depression is thought to be the underlying mechanism of migraine aura. In 2006, three relatives having the point mutation E700K in ATPIA2 exon 15 were diagnosed with familial hemiplegic migraine 2 characterized by complicated forms of aura. Here, we generated a transgenic mouse model having the human E700K mutation in the Atpla2 orthologous gene.

Objective: To investigate the characteristics of cortical spreading depression in a mouse model with E700K mutation in the Atpla2.

Methods: Cortical spreading depression was induced by applying stepwise increases of $\mathrm{KCl}$ concentration or electrical stimulation intensity to $\mathrm{C} 57 \mathrm{BL} / 6 \mathrm{~J}-\mathrm{Tg}$ (Atpla2*E700K)915IKwk mice ( $\mathrm{Tg}$, both sexes) and corresponding wild-type animals. Under urethane anesthesia, the responsiveness and threshold to cortical spreading depression were examined and the distribution of c-Fos expression, a neuronal activity marker, was immunohistochemically determined.

Results: Overall, $\mathrm{Tg}$ mice showed significantly faster propagation velocity $(p<0.0 \mathrm{I})$ and longer full-width-at-halfmaximum $(p<0.01)$ than wild-type animals, representing a slower recovery from direct current potential deflection. The cortical spreading depression threshold tended to be lower in $\mathrm{Tg}$, especially in females. c-Fos-positive cells were significantly enhanced in the ipsilateral somatosensory cortex, piriform cortex, amygdala and striatum (each $p<0.05$ vs. contralateral side). Numbers of c-Fos positive cells were significantly higher in the ipsilateral amygdala of Tg, as compared with wild-type animals $(p<0.01)$.

Conclusion: The effect of cortical spreading depression may be greater in E700K transgenic mice than that in wild-type animals, while the threshold for cortical spreading depression shows little change. Higher c-Fos expression in the amygdala may indicate alterations of the limbic system in $\mathrm{Tg}$, suggesting an enhanced linkage between cortical spreading depression and amygdala connectivity in familial hemiplegic migraine 2 patients.
\end{abstract}

\section{Keywords}

$\mathrm{Na}^{+}, \mathrm{K}^{+}$-ATPase $\alpha 2$ subunit, cortical spreading depression, familial hemiplegic migraine 2, c-Fos, E700K mutation

Date received: 27 November 2019; revised: 15 March 2020; accepted: 17 April 2020

\footnotetext{
'Department of Neurology, Keio University School of Medicine, Tokyo, Japan

${ }^{2}$ Department of Neurology, Daping Hospital, Third Military Medical University, Chongqing, China

${ }^{3}$ Division of Biology, Center for Molecular Medicine, Jichi Medical University, Shimotsuke, Japan
}

${ }^{4}$ Division of Physiology, International University of Health and Welfare, Narita, Japan

${ }^{5}$ Department of Neurology, Shonan Keiiku Hospital, Fujisawa, Japan

Corresponding author:

Miyuki Unekawa, Keio University School of Medicine, Department of Neurology, 35 Shinanomachi, Shinjuku-ku, Tokyo 160-8582, Japan.

Email: unekawa.m@z5.keio.jp 


\section{Introduction}

One-third of migraine headache patients experience aura with transient focal neurological abnormalities such as visual or sensory deficits (1). The pathophysiological mechanism underlying migraine aura is thought to be cortical spreading depolarization/depression (CSD) $(2,3)$, which is a wave of massive depolarization of neurons and glial cells (4). It is postulated that meningeal inflammation and trigeminal sensitization induced by CSD may be responsible for the development of migraine headaches (5).

Migraine is a multifactorial episodic brain disorder with a hereditary background $(6,7)$. For most multifactorial diseases, examination of rare monogenic forms showing at least some of the usual clinical features can be helpful to understand the molecular and cellular mechanisms involved. Monogenic autosomal dominantly inherited subtypes of migraine are known as familial hemiplegic migraine (FHM), characterized by complicated forms of migraine aura, including motor weakness, and at least one first- or second-degree relative with migraine aura including motor weakness (8). So far, three subtypes of FHM, termed FHM1-3, have been identified, each caused by distinctive mutations in a specific gene (9-11). In FHM2, a mutation in the ATP1A2 gene, which codes the $\alpha 2$ subunit of $\mathrm{Na}^{+}$, $\mathrm{K}^{+}$-ATPase expressed in astrocytes, may be related to reduced $\mathrm{Na}^{+} / \mathrm{K}^{+}$pumping function, resulting in impaired $\mathrm{K}^{+}$clearance and/or glutamate reuptake, which may induce depolarization of adjacent neurons and reduce the activation threshold $(12,13)$.

Animal models with genetic mutations have been used to gain insight into the pathophysiology of FHM. Mouse models of FHM2 harboring the W887R or G301R mutation in the Atpla2 gene exhibit increased susceptibility to CSD $(14,15)$. Higher SD susceptibility was also observed in acute brain slices from $\alpha 2$ heterozygous mice (16). Cell cultures and patchclamp recordings showed reduced rates of glutamate and $\mathrm{K}^{+}$clearance by cortical astrocytes and reduced density of GLT-1a glutamate transporters in perisynaptic astrocytic processes, which may enhance the sensibility to CSD in FHM2 (17,18). Spontaneous "plumes" of glutamate signaling in FHM2 may be a new mechanism not only for migraine, but also potentially other disorders in which SD occurs (19).

We previously demonstrated a low threshold and high propagation velocity for CSD in two Atpla2-defective heterozygous mouse strains (20), Atpla2 $^{\text {tm1Kwk }}(\mathrm{C}-\mathrm{KO})(21)$ and $\operatorname{Atpla2}^{\text {tm2Kwk }}$ (N-KO) (22), in which the site of deletion is different. We found a difference in susceptibility between C-KO and N-KO mice. We speculate that the phenotype difference between them depends upon the residual gene function, such as dominant negative effect.

The single base change E700K mutation in ATP1A2 exon 15 , which replaces glutamic acid for lysine, was segregated in three affected Italian individuals from one family in 2006 (23). The mutation may affect a highly conserved residue among $\mathrm{Na}^{+} / \mathrm{K}^{+}$and $\mathrm{H}^{+} / \mathrm{K}^{+}$ pumps, and produces charge difference in the intracellular loop, which contains important regulatory domains for the ion transport system (23). It is also possible that the mutation may hinder the correct biosynthesis of $\mathrm{Na}^{+}, \mathrm{K}^{+}$-ATPase, which could account for the neurological disorder in FHM2. The E700Kmutated FHM2 patients showed a stereotypic pattern of migrainous headache and other neurological symptoms including long-lasting hemisensory and hemiparetic attacks, blurred vision, and speech disturbance during aura episodes (23).

To investigate how a single amino acid substitution affects neurological function in vivo, we examined the threshold for CSD and the characteristics of CSD elicitation in E700K transgenic mice $(\mathrm{Tg})$ and their wild-type littermates (WT). Moreover, the distribution of c-Fos expression, as a marker of neuronal excitation, after CSD induction was immunohistochemically determined to examine the regions caused by CSD.

\section{Materials and methods}

\section{Animals}

All experiments were performed with the approval (No.09058) of the Animal Ethics Committee of Keio University (Tokyo, Japan) in accordance with the university's guidelines and the ARRIVE guidelines (animal research: reporting in vivo experiments) for the care and use of laboratory animals. Data were obtained from 9- to 11-week-old male and female heterozygous C57BL/6J-Tg(Atp1a2*E700K)9151Kwk mice $(\mathrm{Tg}$; male $\mathrm{n}=24$, female $\mathrm{n}=28$ ) and their wildtype littermates (WT; male $n=32$, female $n=28$ ). All the mice were housed in dedicated temperature- and humidity-controlled rooms $\left(23.0 \pm 1.0^{\circ} \mathrm{C}, \quad 55 \pm 7 \%\right)$ with food and tap water ad libitum.

\section{Generation of E700K transgenic mice}

We constructed BAC harboring the point mutation E700K in the Atpla2 locus by using a Counterselection BAC modification kit (Gene Bridges $\mathrm{GmbH}$, Heidelberg, Germany). Detailed methods including the sequences of primers used are described in Table 1 . Briefly, the Atpla2 fragment having the E700K (A2098G) mutation was prepared by site-specific mutagenesis. The mutated BAC DNA was used for 
Table I. Primers used in the construction of E700K mutant mice.

\begin{tabular}{lllll}
\hline $\begin{array}{l}\text { Primer } \\
\text { name }\end{array}$ & Product & Position & Sequence & Alternate name \\
\hline PIF & r-K cassette & Forward & CAggatccTCAGAAGAACTCGTCAAGAAGGCGATAG & rpsL-neoF-EcoRI \\
P2R & r-K cassette & Reverse & CAgaattcGGCCTGGTGATGATGGCGGGATC & rpsL-neoR-BamHI \\
P3F & Left arm & Forward & GGCGGCCGCtctagaCTGTGTCACCTTGGAAACATCTTGCAG & a2-700LF-Xbal \\
P4R & Left arm & Reverse & CTTCTGAggatcCTgCTCTGACACCCTTCCACAATGATAAGC & a2-700LR-BamHI \\
P5F & Right arm & Forward & CCAGGCCgattcTgGCTTATCATTGTGGAAGGGTGTCAGAG & a2-700RF-EcoRI \\
P6R & Right arm & Reverse & GGGAACAAAAGCTGggtaccGAGGTTCTGAGGGCATCAGGGAG & a2-700RR-Kpnl \\
P7R & Mutagenesis & Reverse & GAGGTCCGGGCAAAGACAATCTCCGTGTGGTCCCTcAaGATCTC & a2-E700KLR2 \\
P8F & Mutagenesis & Forward & TTTGCCCGGACCTCCCCTCAGCAGAAGCTTATCATTGTGaAAGG & a2-E700KRF2 \\
\hline
\end{tabular}

generation of E700K transgenic mice (24). (Figure 1(a), (b), see Supplemental Material 1 for details).

\section{Identification of estrous cycle of the female mice}

We previously reported that the threshold for CSD is significantly lower during diestrus in C57BL/6J female mice (25). Therefore, the natural estrous cycle of all female mice was determined for at least five consecutive days (once a day), as previously described. Briefly, vaginal mucous membrane cells were stained with Giemsa stain solution and the ratio of nucleated epithelial cells, cornified squamous epithelial cells and leukocytes was evaluated each day. Experiments were done when the estrous stage was determined as proestrus, estrus or metestrus, but not diestrus (Figure 2).

\section{Electrophysiological examination}

The procedures were previously described in detail (20) and are summarized in Figure 3. Briefly, mice were anesthetized with urethane $(1.1 \mathrm{~g} / \mathrm{kg}$ body weight, intraperitoneally) and the body temperature was maintained with a thermally regulated heating pad (BWT-100; Bioresearch Center Co. Ltd., Nagoya, Japan). Each mouse was fixed in a head-holder apparatus (SG-4N, Narishige Scientific Instrument Laboratory, Tokyo, Japan) and artificially respirated through an intratracheal catheter with a small animal ventilator (SAR-830/AP, CWE Inc., Ardmore, PA, USA). The end-tidal $\mathrm{CO}_{2}$ level was maintained around $4 \%$ by adjusting the ventilation volume and rate using a $\mathrm{CO}_{2}$ analyzer (microCapStar, CWE Inc.). Systemic arterial blood pressure (SABP) and heart rate (HR) were recorded with a non-invasive blood pressure monitor (MK-2000ST, Muromachi Kikai Co. Ltd., Tokyo, Japan) through the tail.

The $\mathrm{Ag} / \mathrm{AgCl}$ electrodes (tip diameter $=200 \mu \mathrm{m}$, EEG-5002Ag; Bioresearch Center Co. Ltd.) were fixed on the dura at the parietal cortex $(2 \mathrm{~mm}$ lateral and $2 \mathrm{~mm}$ caudal to the bregma) and the frontal cortex
( $2 \mathrm{~mm}$ lateral and $2 \mathrm{~mm}$ rostral to the bregma). Reference electrodes (EER-5004Ag; Bioresearch Center Co. Ltd.) were subcutaneously placed on the back. Direct current (DC) potential was amplified at $1-100 \mathrm{~Hz}$ and digitized at $1 \mathrm{kHz}$ with a differential headstage and differential extracellular amplifier (Model 4002 and EX1; Dagan Co., Minneapolis, MN, USA). Regional cerebral blood flow (rCBF) was monitored with a laser Doppler flowmetry (LDF, ALF 21R; Advance Co. Ltd., Tokyo, Japan) on the skull at the coordinates of $4 \mathrm{~mm}$ lateral and $2 \mathrm{~mm}$ posterior to the bregma.

\section{Evaluation of CSD threshold}

A small hole was made on the occipital cortex $(2 \mathrm{~mm}$ lateral and $5 \mathrm{~mm}$ posterior to the bregma) for $\mathrm{KCl}$ application. The dura was carefully slightly incised using a 30-gauge needle. When there had been no CSD episode for at least $30 \mathrm{~min}$ and all parameters had been stable for at least $10 \mathrm{~min}$, we dropped a $5 \mu \mathrm{l}$ aliquot of $0.025 \mathrm{M} \mathrm{KCl}$ into the hole. This was followed by further aliquots with stepwise increases of $\mathrm{KCl}$ concentration by $0.025 \mathrm{M}$ at 5 -minute intervals until CSD was induced (Figure 3(b)).

The CSD threshold was also determined by electrical stimulation as a distinct but complementary method. Electrical stimulation was applied through a unipolar electrode (tip diameter $500 \mu \mathrm{m}$ and inner stainless electrode diameter $200 \mu \mathrm{m}$; EKC-2005S-1902, Bioresearch Center Co., Ltd.) placed on the occipital cortical surface with a constant-current stimulus isolator (A385, World Precision Instruments Inc., FL, USA) at 4-minute intervals, and the charge was increased stepwise, varying both the current intensity and frequency. The pulse duration was set to $10 \mathrm{msec}$ and the maximum pulse rate was set to $30 \mathrm{~Hz}$. At $1 \mathrm{~mA}$ current, pulses of 50,100, 200, 300, and $400 \mathrm{msec}$ were applied, followed by $2 \mathrm{~mA}$ current of 300,400 , and 500 $\mathrm{msec}$, then $3 \mathrm{~mA}$ for $400 \mathrm{msec}$, and $4 \mathrm{~mA}$ for 400,500 , 


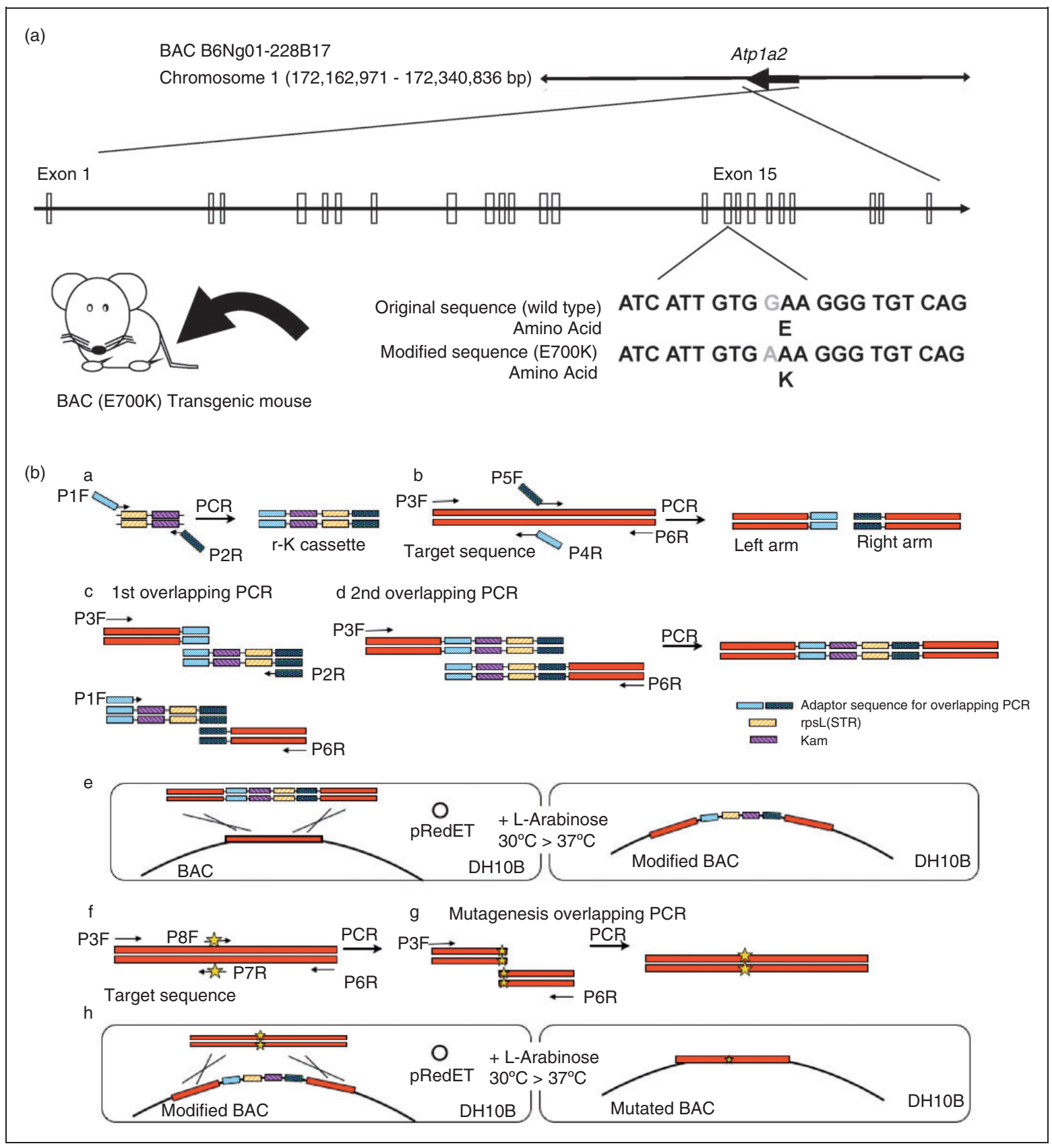

Figure I. Generation of E700K transgenic mice. (a) Targeting strategy for mutating the $\mathrm{Na}^{+}, \mathrm{K}^{+}$-ATPase $\alpha 2$ subunit gene (Atp la2). (b) Construction of E700K transgenic mouse (see Supplemental Material I for details).

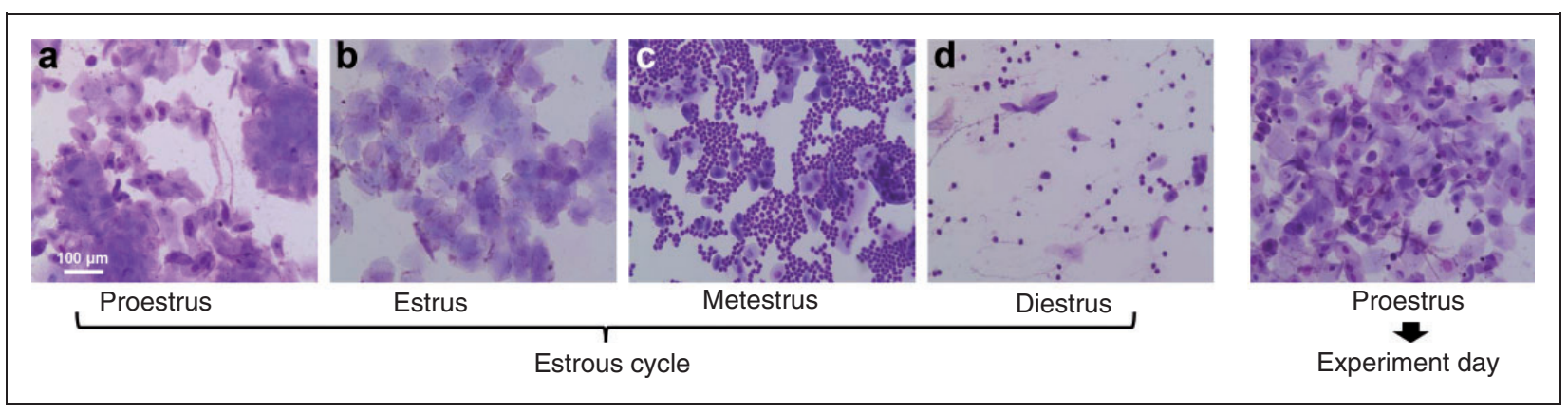

Figure 2. An estrous cycle, based on cytological findings of the vaginal fluid, consists of four distinct stages: (a) proestrus stage, (b) estrus stage, (c) metestrus stage, (d) diestrus stage. The condition on the day of experiment is shown on the right. 


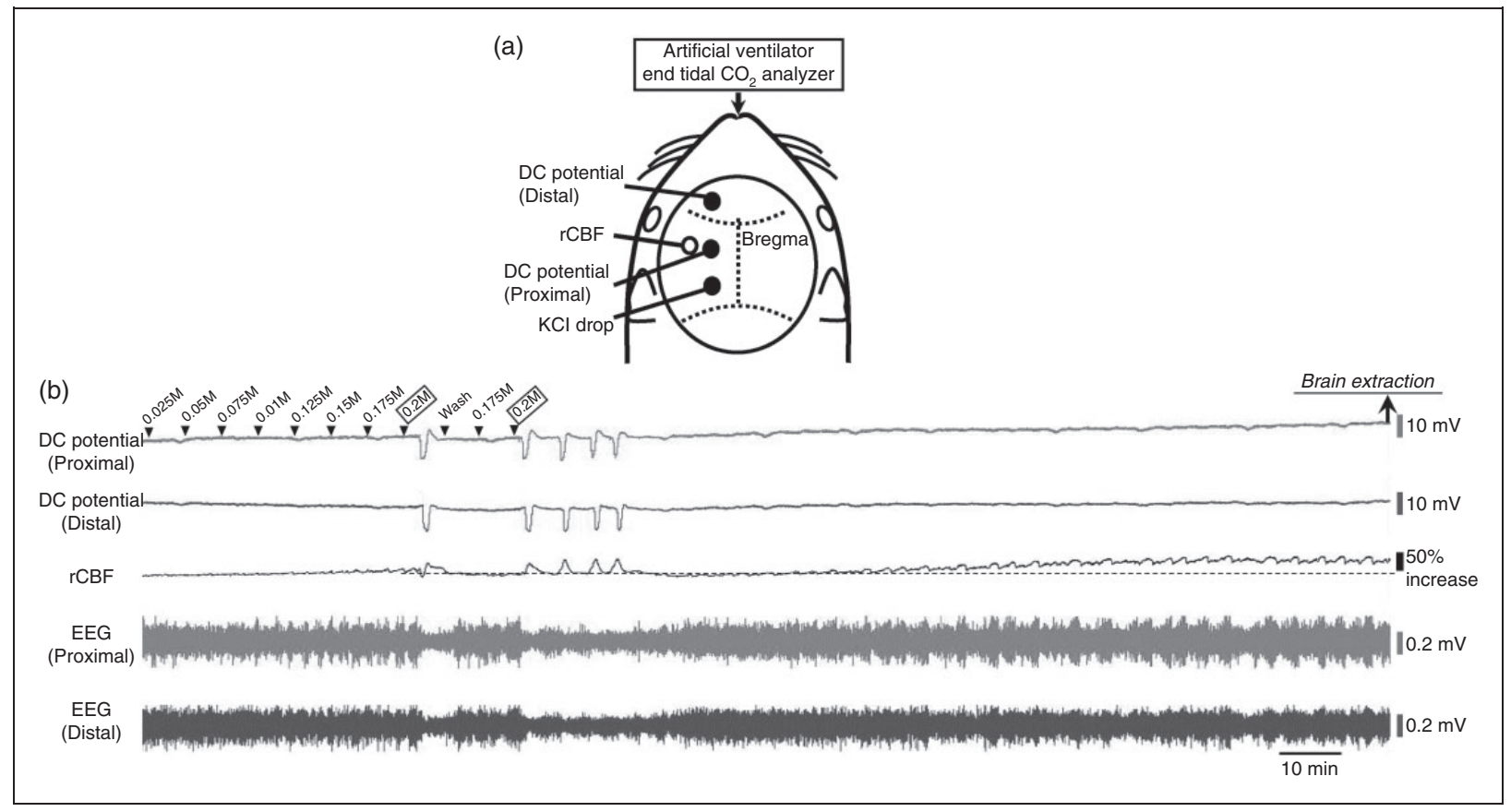

Figure 3. Schematic illustration of this study. (a) Schematic representation of the electrophysiological recording. Diagram showing craniotomy for the placement of DC electrodes, the laser Doppler flowmetry probe, and $\mathrm{KCl}$ application site. (b) Representative example of $\mathrm{DC}$ potential, EEG and $\mathrm{rCBF}$ recordings demonstrating the overall protocol. $\mathrm{KCl}$ was applied in stepwise-increasing concentrations at the arrowheads, and the whole brain was extracted for immunohistochemical examination at 2 hours after CSD induction.

and $1000 \mathrm{msec}$. The applied intensity is shown in microcoulombs $(\mu \mathrm{C})$ by current $(\mathrm{mA}) \times$ time (pulse duration $\times$ repetition numbers, $\mathrm{msec})$. The stimulation protocol was terminated when a CSD occurred. If CSD did not occur, the threshold was assumed as the highest charge assigned (26).

The concentration of $\mathrm{KCl}$ or the applied intensity of electrical stimulation at which CSD first occurred, demonstrating a distinct DC potential deflection, typical fluctuation of $\mathrm{rCBF}$ and propagation to the distal portion, was considered as the CSD threshold. The same procedure was then repeated, starting from a lower concentration/intensity than the previous threshold, and the average value of the two trials was calculated and taken as the threshold for the individual mouse.

\section{Immunohistochemistry and quantification}

After the evaluation of the threshold, three CSDs were additionally induced with $0.3 \mathrm{M} \mathrm{KCl}$, and then the hole was washed with physiological saline to stop CSD induction. At 2 hours, after five episodes of CSD in total, mice were transcardially perfused with $4 \%$ paraformaldehyde (PFA)/phosphate-buffered saline (PBS) and their brains were dissected and further fixed with 4\% PFA/PBS overnight followed by cytoprotection $(30 \% \mathrm{w} / \mathrm{v}$ sucrose in PBS). Whole brains were embedded in optimal cutting temperature compound, frozen in liquid nitrogen, cut into $30 \mu \mathrm{m}$-thick coronal sections, and mounted on silane-coated glass slides.

We conducted enhanced immunohistochemistry using anti-c-Fos antibody (1:2000, Ab-5, Oncogene Research Products, MA, USA, or ABE457, Merck KGaA, Darmstadt, Germany) as the first antibody, fluorescent conjugated anti-rabbit antibody (1:2000, Thermo Fisher Scientific, MA, USA) as the secondary antibody, and alkaline phosphatase (AP)-conjugated anti-fluorescent antibody (1:2000, Roche Diagnostics, Basel, Switzerland) as the third antibody. Signals were detected with NBT/BCIP (Roche Diagnostics) as the chromogen. Bluestained c-Fos-immunoreactive nuclei were regarded as c-Fos-positive cells. Anatomical positions were detected relative to the bregma using Paxinos and Franklin's atlas of the mouse brain (27). The following brain regions were counted and results were calculated as the average of two consecutive sections: The somatosensory cortex, $0.5 \mathrm{~mm}$ square region around the bregma $-0.70 \mathrm{~mm}$; the striatum, $1 \mathrm{~mm}$ square region around the bregma $0.70 \mathrm{~mm}$; the piriform cortex, $500 \mu \mathrm{m}$ length around the bregma $-0.7 \mathrm{~mm}$; the amygdala, $1 \mathrm{~mm}$ square region around the bregma $-1.6 \mathrm{~mm}$. Counting was performed at the same position on the ipsilateral and contralateral sides of each brain. 


\section{Data analysis}

We evaluated CSD profiles as previously reported (20). The time difference and distance between the proximal and distal recording electrodes were used to calculate propagation velocity. Maximum decrease of DC potential and full-width-at-half-maximum (FWHM) were obtained from the distal electrode, because the basal level at proximal electrodes may be influenced by $\mathrm{K}^{+}$ infiltration. We averaged the DC potential and $\mathrm{rCBF}$ every $5 \mathrm{sec}$ using the time point when the value fell below the pre- $\mathrm{KCl}$ average minus twice the standard deviation (SD) as the criterion time. Initial decrease of $\mathrm{rCBF}$ and subsequent increase were determined from the first CSD, because subsequent CSDs showed only an increase. The electroencephalogram (EEG) was evaluated from the DC potential signal by digital filtering with a $5-20 \mathrm{~Hz}$ bandpass, and the root-meansquare value was calculated every $60 \mathrm{sec}$ after the first CSD. DC potential and $\mathrm{rCBF}$ were averaged every 5 sec, and EEG was obtained every $1 \mathrm{~min}$, presented relative to the pre-CSD level, and temporally averaged using the time point when the DC potential began to decrease as the criterion time.

Data are presented as mean $\pm \mathrm{SD}$. The results of each treatment in the Tg group and WT group were compared using a two-tailed Student's t-test after confirming normal distributions according to the KolmogorovSmirnov test. The results of c-Fos expression in the tissues on the operated and contralateral sides were compared using a paired t-test. A $p$ value $<0.05$ was considered statistically significant. All tests were performed with SPSS 23 (IBM, Armonk, NY).

\section{Results}

\section{CSD responsiveness}

The animals' body weights and physiological parameters including HR and SABP showed no significant difference between the Tg and WT (Table 2). A CSD occurred following dura incision in 64\% (9/14) of $\mathrm{Tg}$ and $33 \%(7 / 21)$ of WT among males, and in $56 \%$
(9/16) of Tg and 41\% (7/17) of WT among females. The incidence of CSD occurrence was slightly higher in Tg mice of both sexes. The CSD threshold tended to be lower in Tg than in WT, especially in the female, but not significantly different (female, $p=0.248$; male, $p=0.778$ ) (Figure 4(a)). Propagation velocity in $\mathrm{Tg}$ was significantly faster than in WT (females, $p=0.018$; males, $p=0.021$ ) (Figure 4(b)) and the difference was larger in females (females, $1.04 \mathrm{~mm} / \mathrm{min}$; males, $0.68 \mathrm{~mm} / \mathrm{min}$ ).

Figure 5(a) shows the temporal average of DC potential deflection. Tg showed a significantly larger maximum decrease of DC potential than WT in females $(p=0.016)$, though not in males $(p=0.990)$ (Figure 5 (b)). Meanwhile, FWHM was significantly longer in Tg than in WT among female mice $(p=0.004)$, though not in males $(p=0.155)$ (Figure 5(c)).

EEG was immediately suppressed after first CSD passage, then gradually recovered (Figure 6(a)). The maximum suppression of EEG was similar in $\mathrm{Tg}$ and WT (females, $p=0.954$; males, $p=0.937$ ) (Figure 6(b)).

Similar trends of electrophysiological change were determined in the case of electrical stimulation. The CSD threshold tended to be lower in Tg than in WT, but no significant difference was observed (females, $p=0.450$; males, $p=0.730$ ) (Figure 7(a)). Tg showed a faster propagation velocity than WT in males (females, $p=0.197$; males, $p=0.027$ ) (Figure 7(b)), while the maximum decrease of DC potential was similar in the two strains (females, $p=0.584$; males, $p=0.181$ ) (Figure 7(c)). Meanwhile, FWHM was significantly longer in $\mathrm{Tg}$ in both females and males (females, $p<0.01$; males, $p=0.010$ ) (Figure 7(d)).

\section{Effects on rCBF}

During passage of the first CSD wave, characteristic rCBF changes were observed: An initial decrease, subsequent increase and long-lasting decrease (Figure 8 (a)). The initial decrease of $\mathrm{rCBF}$ was similar between the genotypes in both male and female mice (females, $p=0.203$; males, $p=0.649$ ), whereas the subsequent increase was significantly larger in $\mathrm{Tg}$ male mice

Table 2. Physiological parameters before/after CSD induction by $\mathrm{KCl}$.

\begin{tabular}{|c|c|c|c|c|c|c|}
\hline \multirow[b]{2}{*}{ Group } & \multirow[b]{2}{*}{$\begin{array}{l}\text { Body weight } \\
\text { (g) }\end{array}$} & \multirow[b]{2}{*}{$\mathrm{n}$} & \multicolumn{2}{|c|}{ Before CSD } & \multicolumn{2}{|c|}{ After CSD } \\
\hline & & & $\begin{array}{l}\text { SABP } \\
(\mathrm{mmHg})\end{array}$ & $\begin{array}{l}\mathrm{HR} \\
(\mathrm{bpm})\end{array}$ & $\begin{array}{l}\text { SABP } \\
(\mathrm{mmHg})\end{array}$ & $\begin{array}{l}\mathrm{HR} \\
(\mathrm{bpm})\end{array}$ \\
\hline Male-Tg & $26.0 \pm 1.5$ & 14 & $76 \pm 19$ & $587 \pm 65$ & $82 \pm 17$ & $575 \pm 52$ \\
\hline Male-WT & $26.1 \pm 1.2$ & 21 & $78 \pm 9$ & $558 \pm 57$ & $81 \pm 10$ & $502 \pm 51$ \\
\hline Female-Tg & $21.3 \pm 4.2$ & 16 & $68 \pm 8$ & $659 \pm 32$ & $72 \pm 10$ & $637 \pm 47$ \\
\hline Female-WT & $21.5 \pm 1.3$ & 17 & $71 \pm 9$ & $648 \pm 52$ & $7 I \pm 8$ & $619 \pm 29$ \\
\hline
\end{tabular}

SABP: systemic arterial blood pressure; HR: heart rate. 




Figure 4. Properties of CSD induction. (a) CSD threshold defined as the minimum potassium concentration required to evoke CSD. (b) Propagation velocity calculated from the time difference and distance between the proximal and distal DC recording electrodes. Black columns show transgenic mice ( $\mathrm{Tg}$ ) and gray columns show wild-type mice (WT); filled columns show females, and hatched columns show males. The error bars show standard deviations. $p$-values between Tg and WT were evaluated with a two-tailed t-test and asterisks $(*)$ indicate statistically significant differences $(p<0.05)$.

NS: not significant.

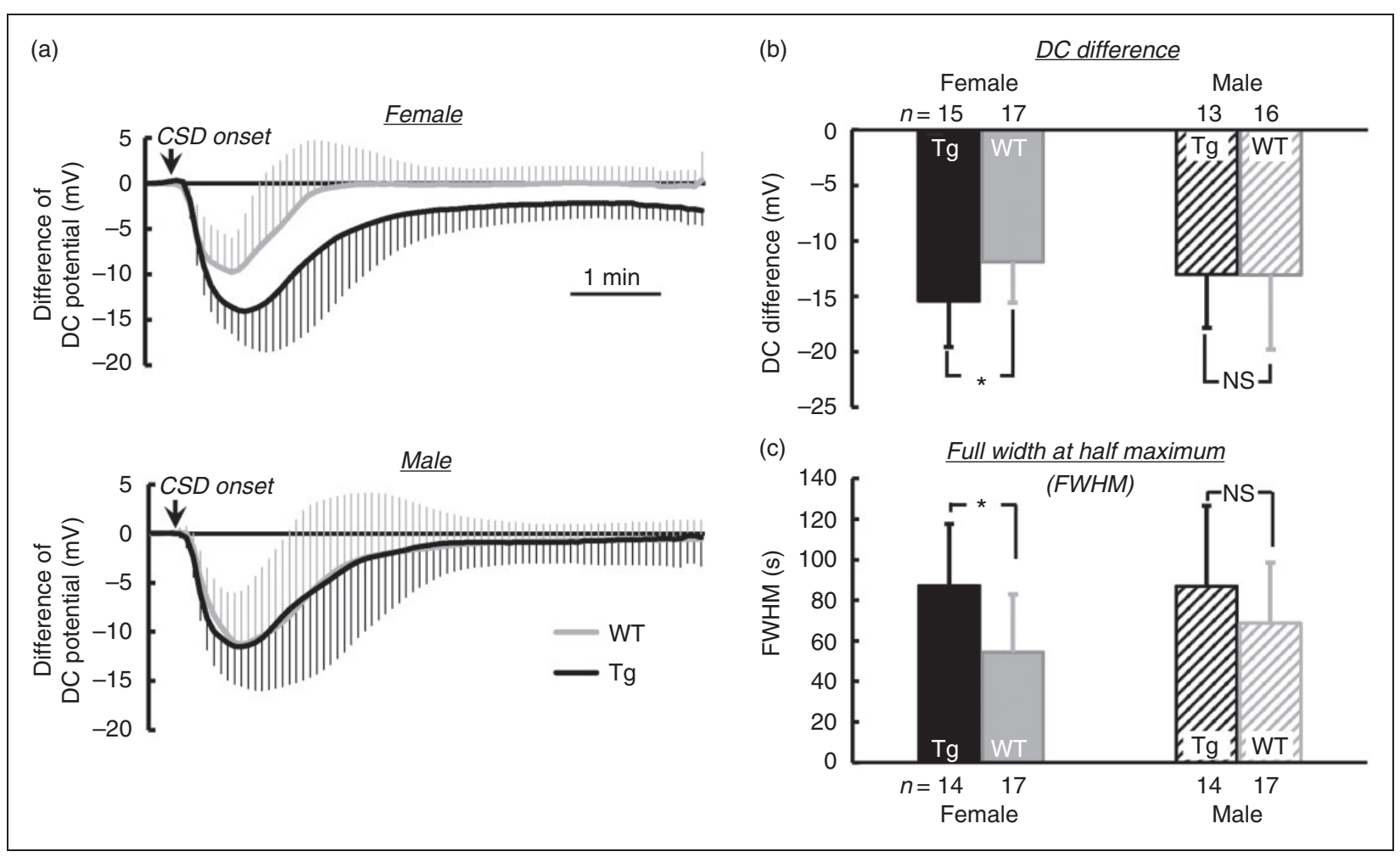

Figure 5. Electrophysiological properties evaluated with DC potential recordings associated with first CSD. (a) Temporal average of DC potential for every $5 \mathrm{sec}$ recorded at the distal electrode. Time 0 was set at the beginning of the DC potential decrease. Tg are indicated in black and WT in gray, and error bars show standard deviations. (b) The maximum change of DC potential deflection and (c) FWHM recorded from the curves at the distal electrode. The $p$-values between $\mathrm{Tg}$ and WT were evaluated with a two-tailed t-test and asterisks $\left(^{*}\right)$ indicate statistically significant differences $(p<0.05)$.

NS: not significant. 


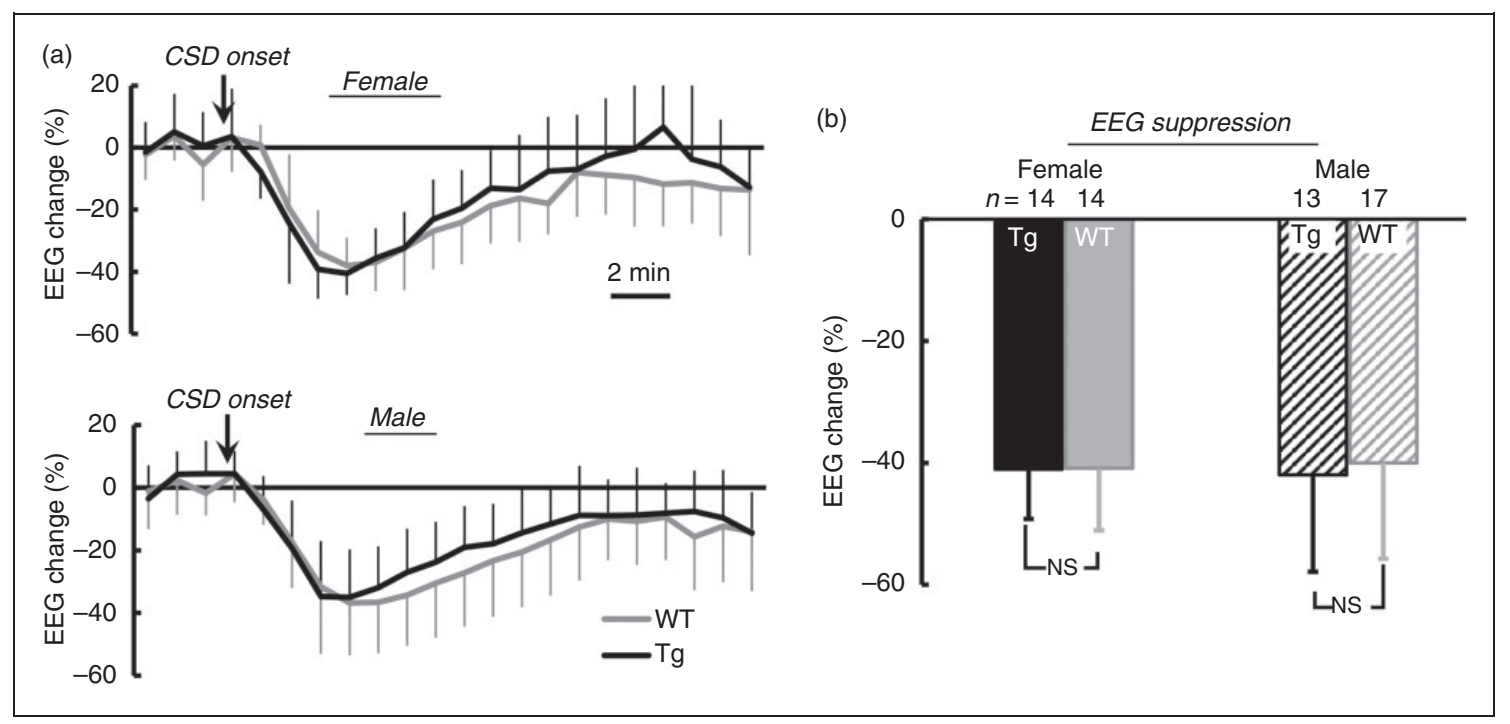

Figure 6. Change of EEG associated with first CSD. (a) Temporal average of EEG for every I min recorded at the distal electrode. (b) The maximal decrease of EEG power determined as the root-mean-square. Tg are indicated in black and WT in gray; filled columns show females and hatched columns show males; error bars show standard deviations. The $p$-values between Tg and WT were evaluated with a two-tailed t-test.

NS: not significant.

(females, $p=0.151$; males, $p=0.034$ ) (Figure $8(\mathrm{~b})$ ). Post-CSD oligemia, represented as basal level before subsequent CSD and transient increase during subsequent CSD, were almost the same; namely, the CSD-induced $\mathrm{CBF}$ changes seem to be comparable (Figure 8(c)). Similar results were obtained in the electrical stimulation test. The initial decrease of $\mathrm{rCBF}$ was similar between the genotypes in both male and female mice (female, $p=0.444$; male, $p=0.154$ ) (Figure 7(e)), whereas the subsequent increase was significantly larger in the $\mathrm{Tg}$ male mice (females, $p=0.231$; males, $p=0.025)$ (Figure 7(f)).

\section{Distribution of c-Fos expression}

c-Fos expression is a validated marker of neuronal activity. We found that c-Fos-positive cells were widespread regions of the ipsilateral side after unilateral cluster episodes of CSD (Supplemental Figure 1 and Supplemental Material 2). Not only the somatosensory cortex, but also essential elements of the limbic system such as the amygdala and piriform cortex, showed robustly enhanced cFos expression, and a small increase was observed in the basal ganglia (striatum) (Figure 9(a), (b)). Expression of c-Fos in the somatosensory and piriform cortex was equally enhanced in the ipsilateral side in Tg and WT (Figure 9(c)). The enhancement of c-Fos expression in $\mathrm{Tg}$ was slightly higher in striatum (females, $p=0.07$; males, $p=0.37$ ), and significantly higher in amygdala (females, $p=0.001$; males, $p=0.001$ ) (Figure 9(c)).

\section{Discussion}

Here we report the first experimental results on CSD in mice carrying the E700K mutation in the Atpla2 gene. The mutation of Glu 700, located in the phosphorylation $(\mathrm{P})$ domain, may affect the salt bridge with lysine 35 , resulting in unstable interactions with the actuator (A) domain. The disruption of domain interactions could be a pathogenic mechanism of $\mathrm{Na}^{+}, \mathrm{K}^{+}$ATPase dysfunction in migraine (28). Various missense mutations in recombinant $\mathrm{Na}$, K-ATPase protein expressed in Sf9 insect cells show heterogeneous affinity for $\mathrm{Na}^{+}$or $\mathrm{K}^{+}$and ATPase activity (28). In kinetic studies of various point-mutated and wild-type ATPases expressed in COS- 1 cells, E700K mutation was not found to interfere with the phosphorylation from ATP, which is known as a critical process for extracellular $\mathrm{K}^{+}$clearance (29). This characteristic may be relevant to our finding that the threshold of CSD occurrence was not affected in our transgenic mice.

It is reported that the reduced CSD threshold in Atpla2 gene-modified mice is due to not only impaired $\mathrm{K}^{+}$clearance by astrocytes, but also reduced glutamate removal from the synaptic cleft, as shown in astrocytes from heterozygous W887R/+ FHM2-knockin mice (17). Reduced function of astrocytes may cause epileptic discharge $(14,30)$. However, in contrast to previously identified FHM2 pedigrees, none of E700K-positive patients reported showed symptom of epileptic seizures (23). Wash-out function of E700K mutant from 


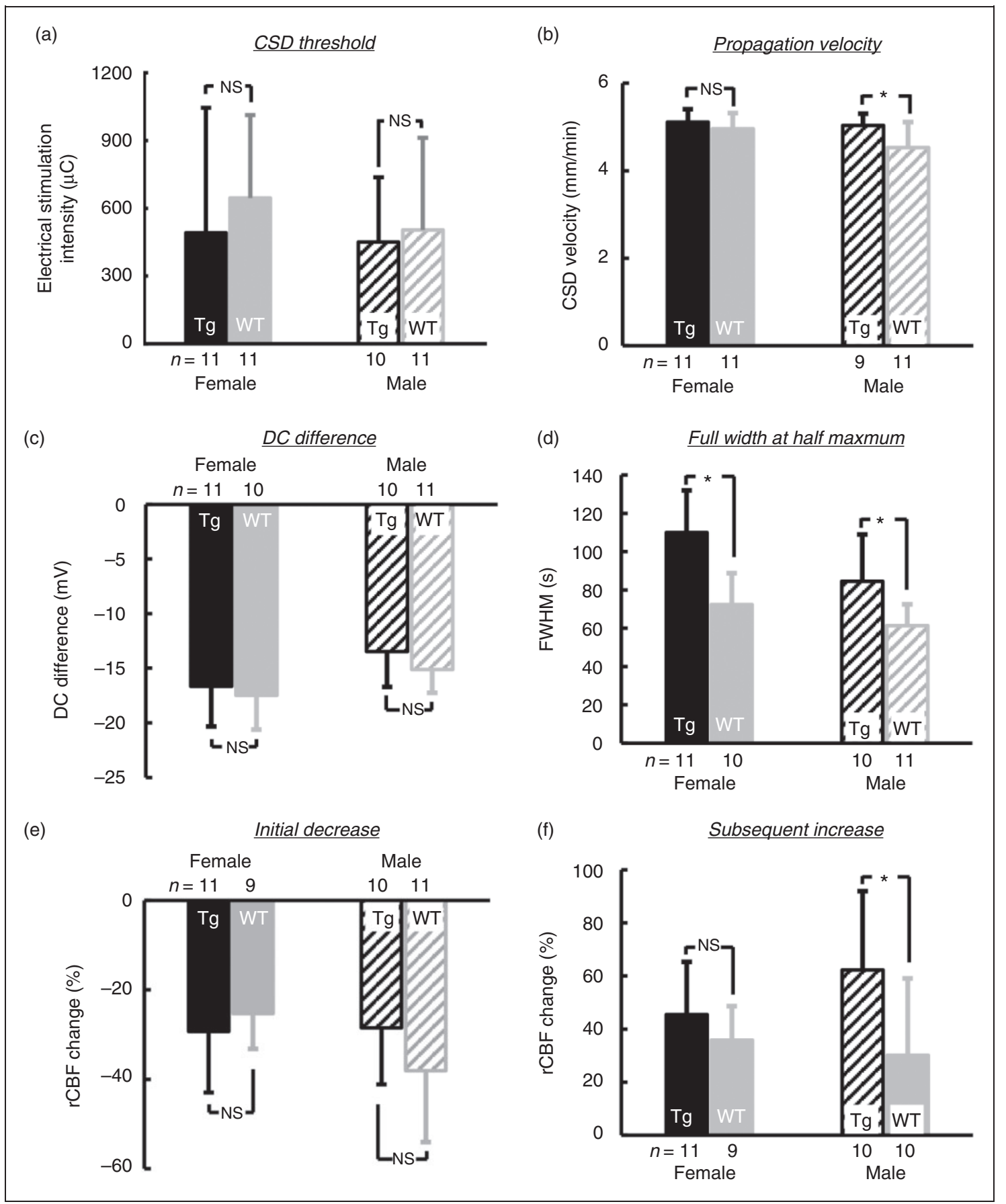

Figure 7. Electrophysiological properties of CSD and rCBF response induced by electrical stimulation. The CSD threshold (a), propagation velocity (b), maximum decrease of DC potential (c), full width at half maximum (FWHM) (d), initial decrease of rCBF (e), and subsequent increase of rCBF (f). Tg are indicated in black and WT in gray. Filled columns show females and hatched columns show males; error bars showed standard deviations. The $p$-values between Tg and WT were evaluated with a two-tailed t-test and asterisks $(*)$ indicated statistically significant differences $(p<0.05)$.

astrocytes may not be drastically reduced compared to other mutants, considered from the kinetic study (29).

Once CSD was induced, both female and male $\mathrm{Tg}$ mice showed higher velocity of CSD propagation.
This could be due to impaired clearance of $\mathrm{K}^{+}$and glutamate by astrocytes consequent to loss-offunction of the pump, resulting in facilitation of CSD spreading by extensive $\mathrm{K}^{+}$and glutamate efflux, which 


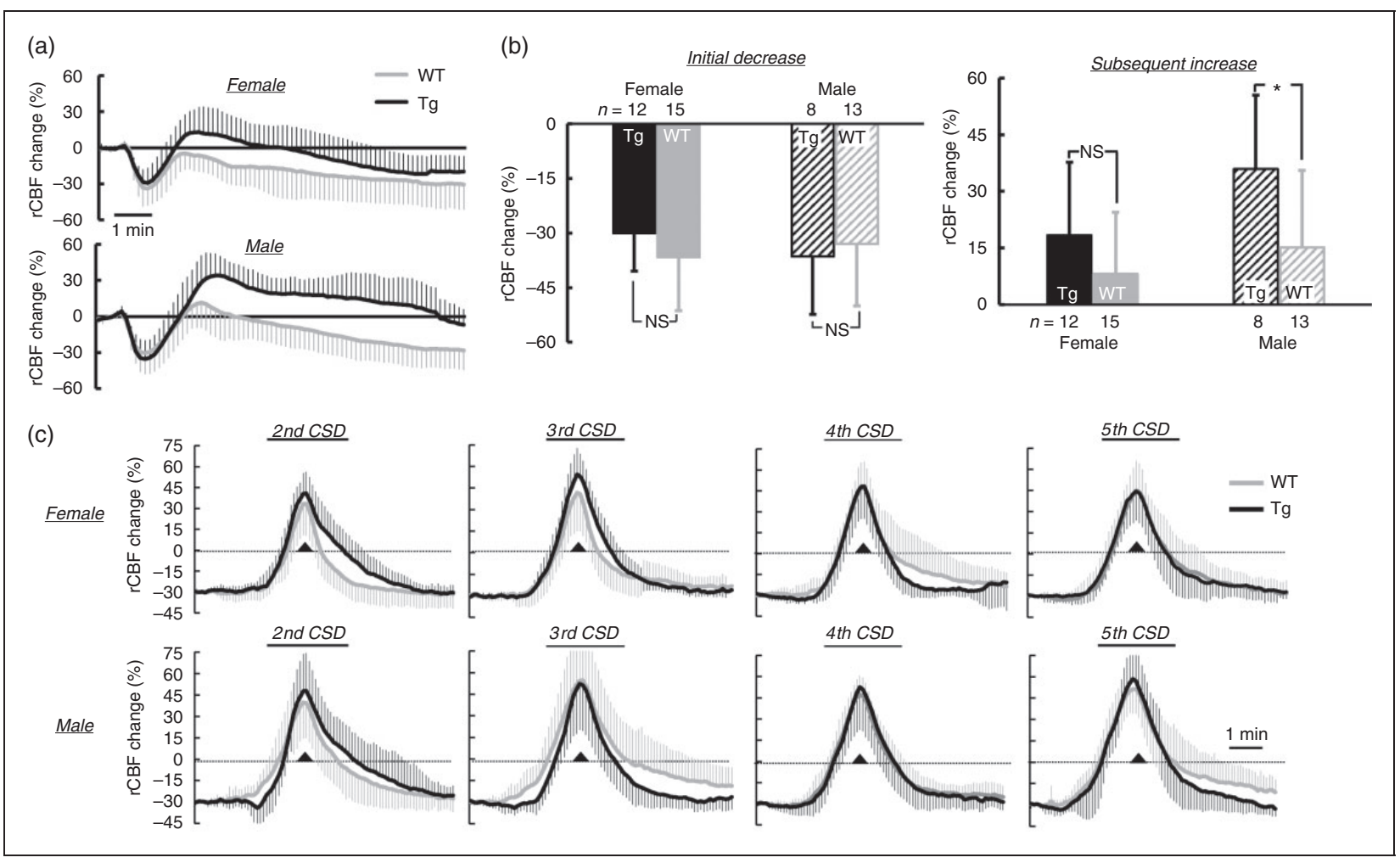

Figure 8. Response of rCBF associated with CSD. (a) Temporal average of rCBF calculated for every $5 \mathrm{~s}$ associated with first CSD. Time 0 was set at the beginning of $\mathrm{rCBF}$ decrease. (b) The maximum change (initial decrease and subsequent increase) of rCBF during passage of the first CSD wave. (c) Temporal change of $\mathrm{rCBF}$ during repetitive CSD after $\mathrm{KCl}$ application. Basal value was fixed at the pre-KCl level for each mouse, indicating post-CSD oligemia. Time 0 was set at the maximum change, as shown by the arrowheads. Tg are indicated in black and WT in gray. Filled columns show females and hatched columns show males; error bars show standard deviations. The $p$-values of differences between $\mathrm{Tg}$ and WT were evaluated with a two-tailed t-test and the asterisk $\left(^{*}\right)$ indicates a statistically significant difference $(p<0.05)$. NS: not significant.

would depolarize adjacent neurons and glia. Some reports have found increased velocity of CSD propagation together with enhanced susceptibility to CSD $(14,15,20,31)$, but the threshold for CSD elicitation was not significantly different in this study. The nature of the relationship is unclear, but the excitability of neurons and glial cells might not always be linked with cellular activity, including ion exchange.

Greater changes of DC potential deflection and delayed recovery from mass depolarization (shown as elongation of FWHM) were seen in female Tg mice. It is well known that migraine is more common in females $(32,33)$. Induction thresholds for CSD are approximately $50 \%$ lower in female mice than males (34). In an animal model of FHM1, female mice were more susceptible to CSD than males, and the susceptibility to CSD might be profoundly affected by sex hormones (35). The results of our study demonstrated a slightly larger difference between $\mathrm{Tg}$ and $\mathrm{WT}$ in female mice for a low threshold of CSD, accelerated propagation, delayed recovery and profound change of DC deflection than male mice. Although no sex-related difference has been reported in other types of FHM2 model mice $(14,15)$, female mice of the present strain may be sensitive to CSD. This phenomenon could be attributed to inherent differences in pain perception and processing of the different genders.

The neuronal suppression (manifested as EEG suppression) and cortical vasoreactivity (manifested as the temporal change and the maximum ratio of $\mathrm{rCBF}$ ) associated with CSD showed no significant difference between $\mathrm{Tg}$ and WT, in accordance with our previous work (20).

The role of CBF in migraine patients remains controversial. Vasodilation of pial and/or meningeal arteries may be associated with pain (36), but the timings of the vasodilation and headache may not necessary correlate (37). Prolonged aura symptoms were frequently associated with hyperperfusion and middle cerebral artery vasodilation in a FHM2 patient (38). Little difference in the rCBF response to CSD was found in Atpla2 heterozygous KO mice in our previous study (20). Interestingly, greater subsequent hyperperfusion, but not initial hypoperfusion, was found in male $\mathrm{Tg}$ mice, suggesting that vasodilation might affect pain sensation. 




Figure 9. Distribution of c-Fos determined immunohistochemically at $2 \mathrm{~h}$ after five consecutive CSDs triggered in a unilateral hemisphere. (a) Coronal sections were taken from three different levels in the whole brain. The white boxes indicate the counting position on the left and the corresponding position on the right. (b) Enlarged photomicrographs showed augmented c-Fos expression in the ipsilateral stimulated hemisphere in the somatosensory cortex, piriform cortex, striatum and amygdala. (c) c-Fos-positive cells counted and averaged over two consecutive sections in the somatosensory cortex, piriform cortex, striatum and amygdala. The data of the ipsilateral hemisphere (i) were compared with the corresponding region of the contralateral hemisphere (c). Tg are indicated in black and WT in gray; filled columns show females, and hatched columns show males. Data are mean $\pm S D$. The $p$-values of differences between $\mathrm{Tg}$ and WT were evaluated with a two-tailed t-test and hash marks (\#) indicate statistically significant differences $(p<0.05)$. The $p$-values of differences between the ipsilateral side and contralateral side were evaluated with a paired t-test and asterisks $(*)$ indicate a statistically significant difference $(p<0.0$ I).

In the present experiments, c-Fos-positive cells were seen in several regions of the ipsilateral hemisphere, including the somatosensory cortex, basal ganglia (striatum) and limbic system (piriform cortex and amygdala), after CSD. This appears to reflect CSD spread into wide cortical and subcortical structures, as already reported in the rat in vitro (39) and mice in vivo (40). Migraine patients show significantly higher blood oxygen level-dependent (BOLD) signal intensities in specific areas including the piriform cortex and limbic structures (amygdala and hippocampus), and higher activity in specific regions including the amygdala and insular cortex in response to olfactory stimulation during migraine attack (41). CSD propagation is affected by the large extracellular space and great abundance of myelin (42). The extracellular $\mathrm{K}^{+}$and glutamate elevation can permeate thinner bundles of myelinate fibers to reignite the process on the other side of the white matter barrier, whereas highly myelinated white matter blocks CSD crossing, therefore only a part of the CSDs might propagate to subcortical structures. Thus, CSD may spread into the deep subcortical tissues of highly susceptible FHM2 patients, such as E700K carriers, and reverberate with cortical and gray matter nuclei. This could result in a severe and prolonged clinical state, including hemiplegia, and coma. In a study of R192Q mutant mice (FHM1), 20-30\% of CSDs propagated into the striatum in WT mice, while over $70 \%$ did so in transgenic mice (40); such propagation may be associated with the prolonged hemiplegia and/or seizure phenotype in migraine with aura.

E700K transgenic mice also showed a stronger ipsilateral c-Fos upregulation in the amygdala than WT, in contrast to the previously examined $\mathrm{N}-\mathrm{KO}$ and C-KO mice (Supplemental Figure 2). The amygdala may play 
an important role in migraine. c-Fos expression was reported in the amygdala, together with behavioral responses consistent with amygdala activation, after a single episode of CSD in freely moving rats (43). Electrophysiological measurement demonstrated that more than $75 \%$ of CSD waves could propagate through the temporal cortex and penetrate to the amygdala in rat slices (39). As a part of the limbic system, the amygdala has functionally essential interactions with these structures and receives highly processed information from the cortex to motor systems, autonomic systems, endocrine systems and other limbic areas associated with emotion, motivation, learning and memory disorders. Clinical studies have shown a lower fractional plasma volume in the left amygdala in migraineurs (44), and the amygdala displays a structural plasticity linked to both headache frequency and the clinical outcome of migraine (45), suggesting that altered functional connectivity of the amygdala and neurolimbic pain network contributes to the pathogenesis of episodic and chronic migraine (46). Furthermore, hyperexcitation and degeneration of the amygdala/piriform cortex in the embryo of homozygotes and enhanced fear/anxiety behaviors in adult heterozygotes were reported in Atpla2-deficient mice (21), suggesting that these limbic systems may be abnormal in some FHM2 model mice. Therefore, we speculate that higher activity in the amygdala due to the propagation of CSD may play an important role in the neurological symptoms during migraine attacks, as indicated by a case report that emotional stress could trigger migraine attack in a patient harboring E700K mutation (23).

In conclusion, the neuro-electrophysiological in vivo evidence for responsiveness to CSD in the present E700K transgenic mice suggests that this mouse model mimics human symptoms, at least in part, and might be useful to study the neuropathology underlying migraine disorders.

\section{Article highlights}

- We report the first experimental results on CSD in mice harboring E700K mutation in the Atp1a2 gene (coding $\mathrm{Na}^{+}, \mathrm{K}^{+}$-ATPase $\alpha 2$ subunit).

- E700K transgenic mice exhibit faster propagation velocity and a longer full-width-at-half-maximum, and Tg mice, especially females, tend to have a lower threshold for CSD initiation, compared to wild-type mice.

- CSD-induced changes in regional cerebral blood flow are comparable in Tg and WT mice.

- c-Fos-positive cells are markedly increased in the CSD ipsilateral side of the somatosensory cortex, piriform cortex, and amygdala, and slightly increased in striatum. The ipsilateral amygdala in $\mathrm{Tg}$ contains more c-Fos positive cells than that in WT.

\section{Declaration of conflicting interests}

The authors declared no potential conflicts of interest with respect to the research, authorship, and/or publication of this article.

\section{Funding}

The authors disclosed receipt of the following financial support for the research, authorship, and/or publication of this article: This work was supported by Keio University Program for the Advancement of Next Generation Research Projects, JSPS KAKENHI (Grant Numbers 19K07849 to MS and 19K17018 to YI) and a research grant from Mitsubishi Tanabe Pharma Corporation. This work was supported in part by Japan China Sasakawa Medical Fellowship (2017816) and State Scholarship Fund of China Scholarship Council (201908500072) to CT.

\section{References}

1. Goadsby PJ, Holland PR, Martins-Oliveira M, et al. Pathophysiology of migraine: A disorder of sensory processing. Physiol Rev 2017; 97: 553-622.

2. Milner PM. Note on a possible correspondence between the scotomas of migraine and spreading depression of Leao. Electroencephalogr Clin Neurophysiol 1958; 10: 705

3. Hadjikhani N, Sanchez del Rio $\mathrm{M}, \mathrm{Wu} \mathrm{O}$, et al. Mechanisms of migraine aura revealed by functional MRI in human visual cortex. Natl Acad Sci USA 2001; 98: 4687-4692.

4. Ayata C. Cortical spreading depression triggers migraine attack: Pro. Headache 2010; 50: 725-730.

5. Costa C, Tozzi A, Rainero I, et al. Cortical spreading depression as a target for anti-migraine agents. J Headache Pain 2013; 14: 62.

6. Sandor PS, Ambrosini A, Agosti RM, et al. Genetics of migraine: Possible links to neurophysiological abnormalities. Headache 2002; 42: 365-377.

7. Tolner EA, Houben T, Terwindt GM, et al. From migraine genes to mechanisms. Pain 2015; 156: S64-S74. 
8. Headache Classification Committee of the International Headache Society (IHS). The International Classification of Headache Disorders, 3rd edition. Cephalalgia 2018; 38 : $1-211$.

9. Ophoff RA, Terwindt GM, Vergouwe MN, et al. Familial hemiplegic migraine and episodic ataxia type-2 are caused by mutations in the $\mathrm{Ca}^{2+}$ channel gene CACNL1A4. Cell 1996; 87: 543-552.

10. Fusco MD, Marconi R, Silvestri L, et al. Haploinsufficiency of ATP1A2 encoding the $\mathrm{Na}^{+} / \mathrm{K}^{+}$ pump $\alpha 2$ subunit associated with familial hemiplegic migraine type 2. Nat Genet 2003; 33: 192-196.

11. Dichgans M, Freilinger T, Eckstein G, et al. Mutation in the neuronal voltage-gated sodium channel SCN1A in familial hemiplegic migraine. Lancet 2005; 366: 371-377.

12. Gritz SM and Radcliffe RA. Genetic effects of ATP1A2 in familial hemiplegic migraine type II and animal models. Hum Genom 2013; 7: 8.

13. Friedrich T, Tavraz NN and Junghans C. ATP1A2 mutations in migraine: Seeing through the facets of an ion pump onto the neurobiology of disease. Front Physiol 2016; 7: 239.

14. Kros L, Lykke-Hartmann $\mathrm{K}$ and Khodakhah $\mathrm{K}$. Increased susceptibility to cortical spreading depression and epileptiform activity in a mouse model for FHM2. Sci Rep 2018; 8: 16959.

15. Leo L, Gherardini L, Barone V, et al. Increased susceptibility to cortical spreading depression in the mouse model of familial hemiplegic migraine type 2. PLoS Genet 2011; 7: e1002129.

16. Reiffurth C, Alam M, Zahedi-Khorasani M, et al. $\mathrm{Na}^{+} /$ $\mathrm{K}^{+}$-ATPase $\alpha$ isoform deficiency results in distinct spreading depolarization phenotypes. $J$ Cereb Blood Flow Metab 2019; 40: 622-638.

17. Capuani C, Melone M, Tottene A, et al. Defective glutamate and $\mathrm{K}^{+}$clearance by cortical astrocytes in familial hemiplegic migraine type 2. EMBO Mol Med 2016; 8: 967-986.

18. Bøttger P, Glerup S, Gesslein B, et al. Glutamate-system defects behind psychiatric manifestations in a familial hemiplegic migraine type 2 disease-mutation mouse model. Sci Rep 2016; 2: 22047.

19. Parker PD, Suryavanshi P, Melone M, et al. Noncanonical glutamate signaling in a genetic model of migraine with aura. Neuroscience. Epub ahead of print 2 January, 2020. DOI: 10.1101/2020.01.02.891770.

20. Unekawa M, Ikeda K, Tomita Y, et al. Enhanced susceptibility to cortical spreading depression in two types of $\mathrm{Na}^{+}, \mathrm{K}^{+}$-ATPase $\alpha 2$ subunit-deficient mice as a model of familial hemiplegic migraine 2. Cephalalgia 2018; 38: $1515-1524$.

21. Ikeda K, Onaka T, Yamakado M, et al. Degeneration of the amygdala/piriform cortex and enhanced fear/anxiety behaviors in sodium pump $\alpha 2$ subunit (Atp1a2)-deficient mice. J Neurosci 2003; 23: 4667-4676.

22. Ikeda K. Malfunction of respiratory-related neuronal activity in $\mathrm{Na}^{+}, \mathrm{K}^{+}$-ATPase 2 subunit-deficient mice is attributable to abnormal $\mathrm{Cl}^{-}$homeostasis in brainstem neurons. J Neurosci 2004; 24: 10693-10701.
23. Pierelli F, Grieco G, Pauri F, et al. A novel ATP1A2 mutation in a family with FHM type II. Cephalalgia 2006; 26: 324-328.

24. Transgenic Mouse Service of Tsukuba University, www. md.tsukuba.ac.jp/LabAnimalResCNT/public_E/TMS. html (accessed 14 November 2019).

25. Ebine T, Toriumi H, Shimizu T, et al. Alterations in the threshold of the potassium concentration to evoke cortical spreading depression during the natural estrous cycle in mice. Neurosci Res 2016; 112: 57-62.

26. Ayata C, Jin H, Kudo C, et al. Suppression of cortical spreading depression in migraine prophylaxis. Ann Neurol 2006; 59: 652-661.

27. Paxinos $G$ and Franklin KBJ. The mouse brain in stereotaxic coordinates. 2nd ed. San Diego: Academic Press, 2001.

28. Swarts HGP, Weigand KM, Venselaar H, et al. Familial hemiplegic migraine mutations affect $\mathrm{Na}, \mathrm{K}$-ATPase domain interactions. Biochim Biophys Acta 2013; 1832: 2173-2179.

29. Schack VR, Holm R and Vilsen B. Inhibition of phosphorylation of $\mathrm{Na}^{+}, \mathrm{K}^{+}$-ATPase by mutations causing familial hemiplegic migraine. J Biol Chem 2012; 287: 2191-2202.

30. Bordey A, Lyons SA, Hablitz JJ, et al. Electrophysiological characteristics of reactive astrocytes in experimental cortical dysplasia. J Neurophysiol 2001; 85: 1719-1731.

31. van den Maagdenberg AM, Pizzorusso T, Kaja S, et al. High cortical spreading depression susceptibility and migraine-associated symptoms in $\mathrm{Ca}(\mathrm{v}) 2.1 \mathrm{~S} 218 \mathrm{~L}$ mice. Ann Neurol 2010; 67: 85-98.

32. Thomsen LL, Eriksen MK, Roemer SF, et al. A population-based study of familial hemiplegic migraine suggests revised diagnostic criteria. Brain 2002; 125: 1379-1391.

33. Thomsen LL, Kirchmann M, Bjornsson A, et al. The genetic spectrum of a population-based sample of familial hemiplegic migraine. Brain 2007; 130: 346-356.

34. Brennan KC, Romero-Reyes M, López Valdés HE, et al. Reduced threshold for cortical spreading depression in female mice. Ann Neurol 2007; 61: 603-606.

35. Eikermann-Haerter K, Baum MJ, Ferrari MD, et al. Androgenic suppression of spreading depression in familial hemiplegic migraine type 1 mutant mice. Ann Neurol 2009; 66: 564-568.

36. Pietrobon D. Migraine: New molecular mechanisms. Neuroscientist 2005; 11: 373-386.

37. Olesen J, Friberg L, Olsen TS, et al. Timing and topography of cerebral blood flow, aura, and headache during migraine attacks. Ann Neurol 1990; 28: 791-798.

38. Iizuka T, Takahashi Y, Sato M, et al. Neurovascular changes in prolonged migraine aura in FHM with a novel ATP1A2 gene mutation. J Neurol Neurosurg Psychiatry 2012; 83: 205-212.

39. Dehbandi S, Speckmann EJ, Pape HC, et al. Cortical spreading depression modulates synaptic transmission of the rat lateral amygdala. Eur J Neurosci 2008; 27: 2057-2065. 
40. Eikermann-Haerter K, Yuzawa I, Qin T, et al. Enhanced subcortical spreading depression in familial hemiplegic migraine type 1 mutant mice. $J$ Neurosci 2011; 31: 5755-5763.

41. Stankewitz A and May A. Increased limbic and brainstem activity during migraine attacks following olfactory stimulation. Neurology 2011; 77: 476-482.

42. Bures J, Buresova O, Krivanek J. The mechanism and applications of Leao's spreading depression of electroencephalographic activity. Prague: Academia, 1974.

43. Akcali D, Sayin A, Sara Y, et al. Does single cortical spreading depression elicit pain behaviour in freely moving rats? Cephalalgia 2010; 30: 1195-1206.
44. Kim YS, Kim M, Choi SH, et al. Altered vascular permeability in migraine-associated brain regions: Evaluation with dynamic contrast-enhanced MRI. Radiology 2019; 292: 713-720.

45. Liu HY, Chou KH, Lee PL, et al. Hippocampus and amygdala volume in relation to migraine frequency and prognosis. Cephalalgia 2017; 37: 1329-1336.

46. Chen Z, Chen X, Liu M, et al. Altered functional connectivity of amygdala underlying the neuromechanism of migraine pathogenesis. J Headache Pain 2017; 18: 7. 